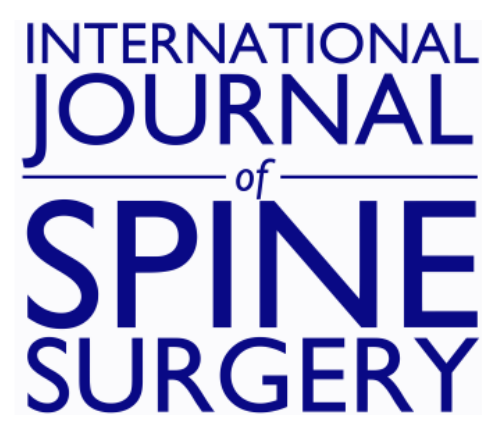

\title{
Is Decompression and Partial Discectomy Advantageous Over Decompression Alone in Microendoscopic Decompression Of Monosegmental Unilateral Lumbar Recess Stenosis?
}

Benedikt W. Burkhardt and Joachim M. Oertel

Int J Spine Surg 2021, 15 (1) 94-104

doi: https://doi.org/10.14444/8013

http://ijssurgery.com/content/15/1/94

This information is current as of April 26, 2023.

Email Alerts Receive free email-alerts when new articles cite this article. Sign up at: http://ijssurgery.com/alerts 


\title{
Is Decompression and Partial Discectomy Advantageous Over Decompression Alone in Microendoscopic Decompression Of Monosegmental Unilateral Lumbar Recess Stenosis?
}

\author{
BENEDIKT W. BURKHARDT, MD, JOACHIM M. OERTEL, MD \\ Department of Neurosurgery, Saarland University Medical Center and Saarland University Faculty of Medicine, Homburg/Saar, Germany
}

\begin{abstract}
Background: Endoscopic techniques are well accepted as surgical technique for decompression of lumbar lateral recess stenosis (LRS). It is uncertain if there is a difference in clinical outcome for decompression alone (DA) or decompression with partial discectomy (DPD) for the treatment of LRS.

Methods: All files of patients who underwent an endoscopic procedure for lumbar LRS were identified from a prospectively collected database. Preoperative magnetic resonance imaging and endoscopic video were analyzed with special focus on the technique of nerve root decompression. Clinical outcome was assessed via a personal examination, a standardized questionnaire including the numeric rating scale (NRS) for leg and back pain, the Oswestry disability index (ODI), and the modified MacNab criteria to assess functional outcome and clinical success.

Results: Sixty-six patients were identified of which 57 attended for evaluation (86.4\%). DA was performed in 15 (26.3\%) patients and DPD in 42 patients (73.7\%). The mean follow-up was 45.0 months (range: $16-82$ months). Fifty-two patients reported to be free of leg pain $(91.1 \%), 42$ patients had no noticeable back pain $(73.7 \%), 49$ patients had full muscle strength $(85.9 \%)$, and 48 patients had no sensory disturbance $(84.2 \%)$. The mean NRS for leg pain was 1 , the mean NRS for back pain was 2, mean ODI was 16\% (range: $0 \%-60 \%$ ). Clinical success was noted in 49 patients $(85.9 \%)$ and it was significantly higher for patients following DPD $(P=.024)$. The overall repeat procedure rate was $12 \%$ with reoperation rate at the index segment in $10.5 \%$ of cases. There were no significant differences with respect to leg and back pain, ODI, and reoperation between both groups.

Conclusion: Microendoscopic DPD of LRS achieves a $92 \%$ clinical success rate which is significantly higher compared to $67 \%$ clinical success achieved by DA. There was no significant difference for the rate of reoperation, leg and back pain, and ODI.
\end{abstract}

Level of Evidence: 4.

Minimally Invasive Surgery

Keywords: decompression, discectomy, endoscopy, lateral recess stenosis, lumbar spine, minimally invasive surgery, tubular retractor

\section{INTRODUCTION}

The degenerative process of the lumbar spine is natural while aging. Degenerative changes of the disc, the facet joint, and the ligamentum flavum are frequently seen in people over the age of 65 years. ${ }^{1}$ The overall incidence for lumbar spinal stenosis (LSS) in the Western world is up to $5.7 \%$. In asymptomatic subjects over 60 years of age a LSS is seen on the magnetic resonance imaging (MRI) scan in $21 \% .^{2}$ Accordingly, LSS is one of the most common indications for surgery in patients older than 60 years. $^{3}$
LSS can occur on the central canal, the nerve root canal, or both. The nerve root canal can be divided in 3 regions: the lateral recess, the neuroforamen, and the extraforaminal part. The lateral recess is confined by several anatomical landmarks. The anterior border is formed by the annulus of the disc and the posterior border is formed by the facet joint. $^{4}$

A stenosis of the lateral recess might be the cause for neurogenic claudication and radicular symptoms such as pain or sensorimotor deficits. ${ }^{5}$ The underlying pathology might be a hypertrophic facet joint with hypertrophic ligamentum flavum, a disc bulge, 
a disc protrusion, or a disc herniation. Once conservative treatment has failed to relief symptoms surgery is recommended for patients. ${ }^{6,7}$ The goal of surgery is to decompress the nerve root beginning from its origin along its course in the lateral recess by preserving spinal stability. A multitude of different surgical techniques have been reported to achieve adequate decompression of the LSS and lumbar lateral recess stenosis (LRS) varying from open to microscopic to tubular endoscopic to shear endoscopic techniques. ${ }^{8-14}$

Full endoscopic and tubular endoscopic procedures have been developed to reduce approachrelated trauma and both techniques have been applied with great success for the treatment of lumbar disc herniation, central canal stenosis, and synovial cyst resection. ${ }^{15-23}$ Endoscopic techniques are well established for the treatment of degenerative disorders of the lumbar spine.

However, surgical treatment of LRS is controversial and there is no consensus on the answer to the question of whether stand-alone decompression is sufficient. The purpose of the present work was to compare the midterm clinical outcome in patients who underwent endoscopic decompression alone (DA) and endoscopic decompression with partial discectomy (DPD) for LRS.

\section{METHODS}

\section{Patient Population and Clinical Evaluation}

A prospectively collected database of endoscopic spine procedures was searched for patients who underwent monosegmental decompression of unilateral lumbar lateral recess stenosis.

Inclusion criteria for this study were the following: no previous lumbar spine surgery, a detailed surgical report that described the technique of LRS decompression, a preoperative MRI that confirms the finding of a LRS, complete endoscopic video recording of the procedure, a complete set of preoperative and postoperative evaluations including a detailed neurological examination, and a minimum follow-up of 12 months.

Retrospectively, all patients' files were reviewed with respect to clinical outcome. The video recording was analyzed with special focuses on the surgical technique for decompression (DA versus DPD), the mobility of the dura and exiting nerve root, and the presence of epidural adhesive tissue.
Table 1. Modified MacNab criteria.

\begin{tabular}{ll}
\hline Rating & \multicolumn{1}{c}{ Definition } \\
\hline $\begin{array}{l}\text { Excellent } \\
\text { Good }\end{array}$ & $\begin{array}{c}\text { Complete resolution of symptoms } \\
\text { Marked improvement but occasional pain which does } \\
\text { not limit the quality of life } \\
\text { Fair }\end{array}$ \\
$\begin{array}{c}\text { Some improvement with the need for pain medications } \\
\text { and significant restrictions in physical activities }\end{array}$ \\
Poor & $\begin{array}{r}\text { No improvement, or worse as compared with the } \\
\text { condition before operation }\end{array}$ \\
\hline
\end{tabular}

Two groups were created (DA and DPD) and compared with respect to their clinical outcome. A personal neurological examination and a standardized questionnaire were conducted which included the assessment of pain level for leg and back pain, Oswestry disability index (ODI) ${ }^{24}$ and functional outcome according to modified MacNab criteria. ${ }^{25}$ Clinical success was defined as excellent and good result according to MacNab criteria (Table 1).

Study design was approved by a local ethical committee (IRB number: 149/17) and patient consent was obtained.

\section{Surgical Technique and Selection Process for DA and DPD}

All procedures were performed under general anesthesia. The patient was placed in a prone position and the diseased segment was identified via a spinal tap needle and lateral fluoroscopy. A paramedian skin incision and opening of the fascia was followed by dilation of the paraspinal muscles. An appropriate sized tubular retractor was inserted with a trajectory perpendicular to the lamina and fixed in its position via a holding arm. A highdefinition endoscope was then inserted in to the tubular retractor and fixed in its position. From this moment on the entire procedure was performed under continuous endoscopic visualization in bimanual microsurgical fashion.

In all procedures the EasyGO spine system (Karl Storz Company, Tuttlingen, Germany) was used. A detailed account of the surgical technique for endoscopic decompression has been reported in detail elsewhere. ${ }^{17,26,27}$ Once the exiting nerve root was identified decompression continued towards the neuroforamen until no nerve root compression was evident.

In case of DA, presence of adhesive epidural tissue which would impede a complete mobilization of the nerve root required performing further dissection and resection of adhesive scare tissue. The decompression was sufficient once the nerve 


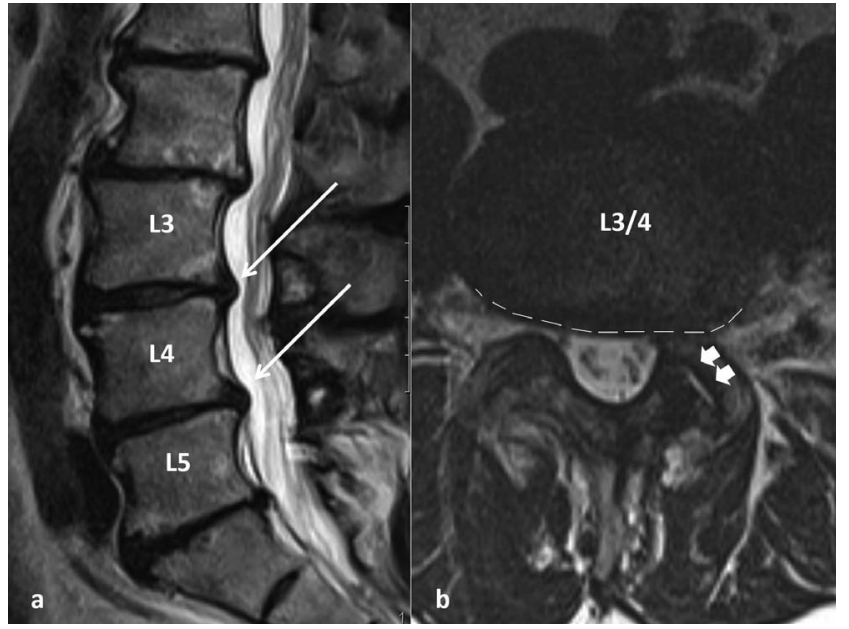

Figure 1. (a) Sagittal T2-weighted magnetic resonance image (MRI) showing a disc bulge (white arrows) at the levels of L3/4 and L4/5. (b) Axial T2-weighted MRI of the segment L3/4 showing a disc bulge (dashed line) with consecutive left-sided lateral recess stenosis and a slight facet joint effusion on the left side (arrowheads).

root was completely mobile and there was no sign of compression or adhesive tissue circumferential to the nerve root, which was inspected via a nerve hook.

DPD was performed once the surgeon could not easily probe the ventral aspect of the nerve root and once there was still tension on the exiting nerve root. DPD was performed using sharp instruments such as a knife to open the posterior longitudinal ligament. This was followed by resection of the annulus and disc material via grasping forceps.

\section{Preoperative MRI Evaluation}

A disc bulge was considered a protrusion of more than $25 \%$ of the disc circumference, patients with this finding were allocated to group 1 (see Figure 1). A disc protrusion was defined as when the disc material extends beyond its normal margin and the margin of the vertebra but involves less than $25 \%$ of the disc circumference, patients with this finding were allocated to group 2 (see Figures 2 and 3). An extrusion of a disc material was defined as the displaced disc material not being in continuity with the disc space. Patients with mediolateral subligamentous dis herniation were allocated to group 3, and in case of partial extrusion of the disc material they were allocated to group 4 (see Figure 4). ${ }^{28,29}$

Hypertrophy of the facet joint and ligamentum flavum with compression of the dura and nerve root, and facet joint effusion were assessed (see Figures 1 and 4). Furthermore, disc degeneration was assessed graded according to Pfirrmann et al. ${ }^{30}$

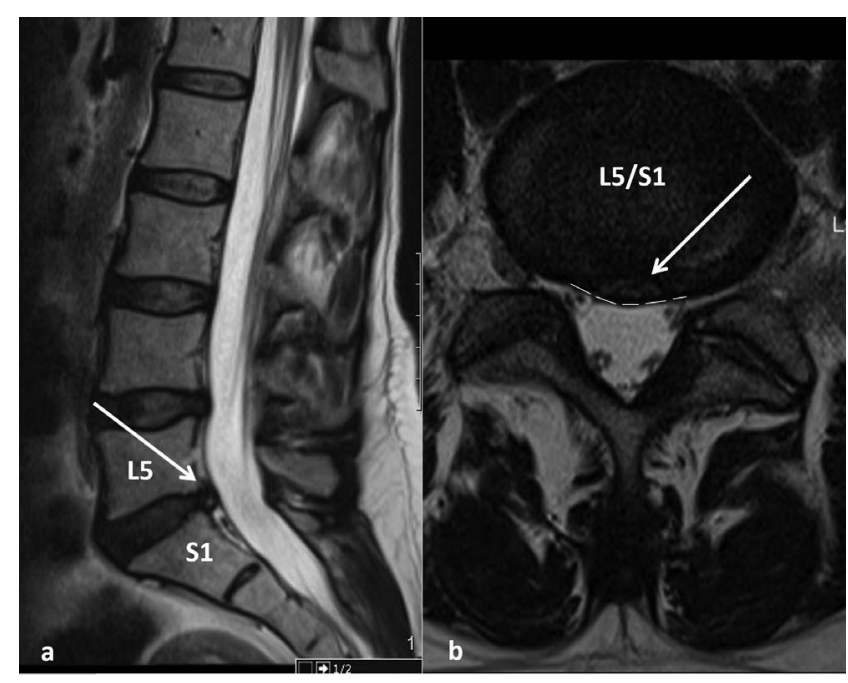

Figure 2. (a) Sagittal T2-weighted magnetic resonance image (MRI)showing a disc protrusion (white arrow) at the level of L5/S1. (b) Axial T2-weighted MRI of the level L5/S1 showing a central (dashed line) disc protrusion (white arrow) with left-sided lateral recess stenosis.

\section{Statistical Methods}

The SPSS statistical software package (SPSS version 25, IBM, Chicago, Illinois) was used for statistical analysis of the data. Two groups were created according to the performed surgical technique (DA and DPD) and clinical outcome, as well as repeated procedures, were compared.

We used 2-sided Fisher exact test to compare relative frequencies of a binary outcome between 2 independent groups. A Wilcoxon test was used to compare nonparametric paired sample tests. Any $P$ values given were 2-sided; $P<.05$ was assumed to be sufficient to indicate statistical significance.

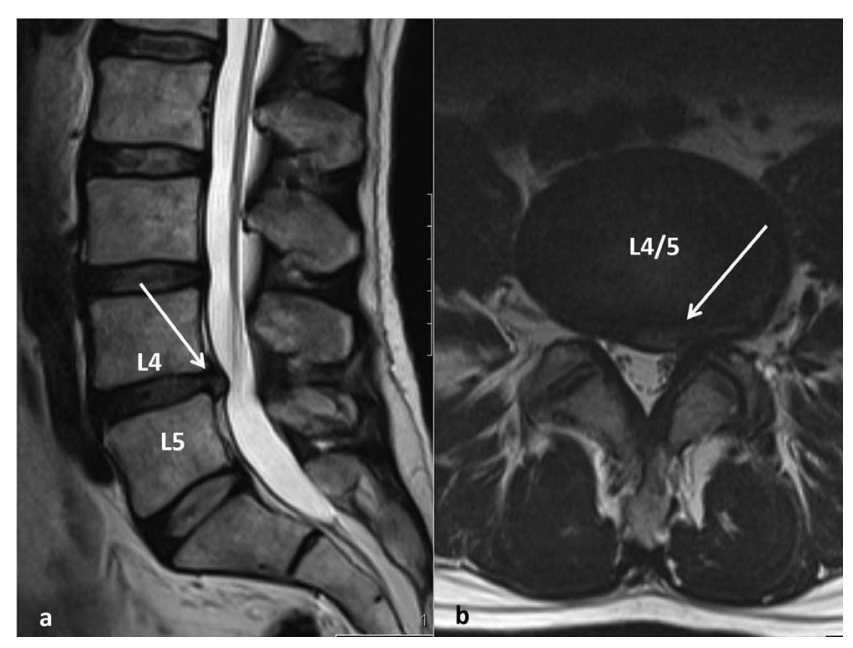

Figure 3. (a) Sagittal T2-weighted magnetic resonance image (MRI) showing a disc protrusion (white arrow) at the level of L4/5. (b) Axial T2-weighted MRI of the level L5/S1 showing a subligamentous mediolateral disc protrusion (white arrow) with left-sided lateral recess stenosis. 


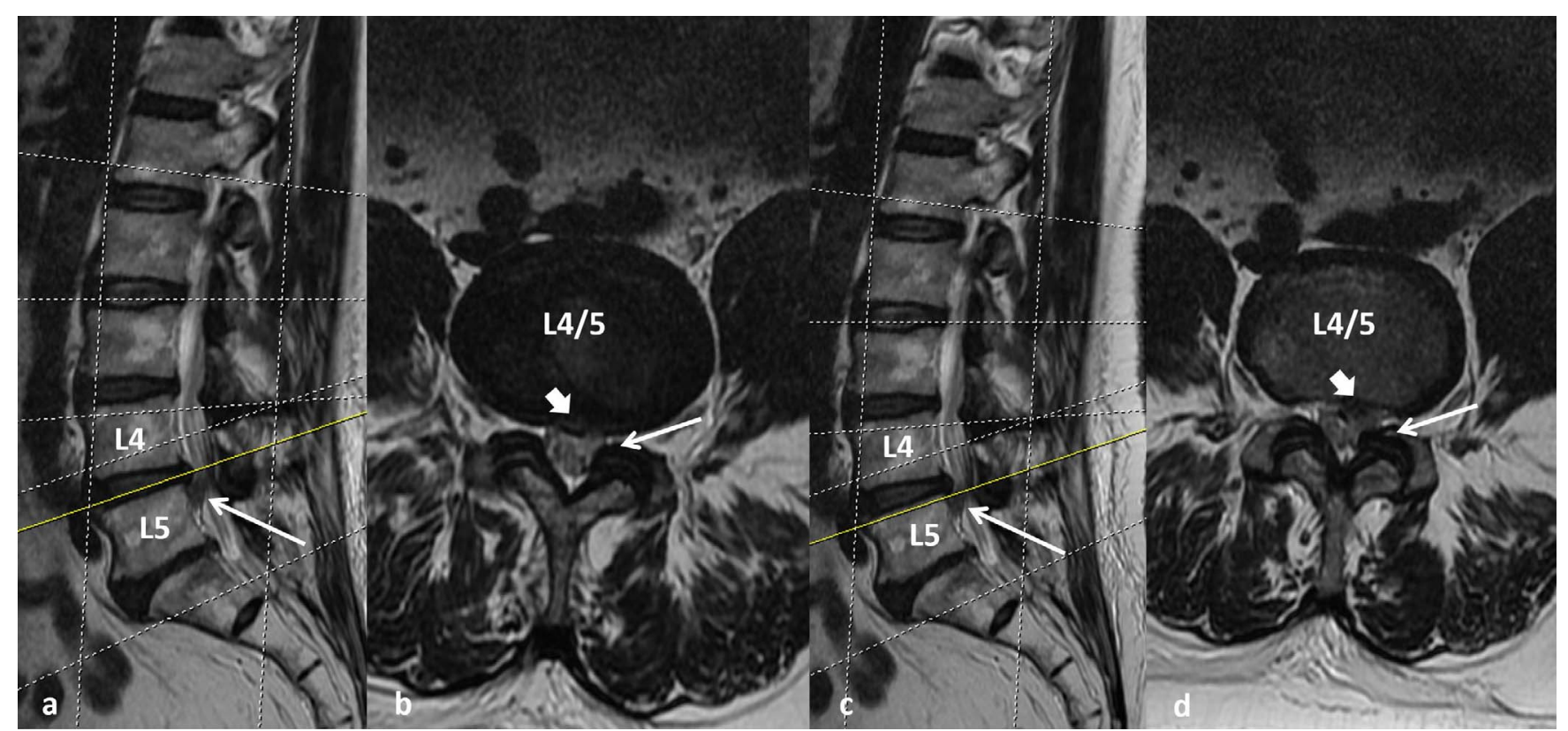

Figure 4. (a, c) Sagittal T2-weighted magnetic resonance images (MRIs) showing an extruded disc fragment (white arrow) caudal to the L4/5 disc space. (b, d) Axial T2-weighted MRIs of the level L4/5 showing a hypertrophic ligamentum flavum (white arrow) and a central subligamentous disc protrusion (white arrowhead) with an extruded fragment and left-sided lateral recess stenosis.

\section{RESULTS}

\section{Patient Characteristics and Intraoperative Findings}

Between 2011 and 2017, 66 patients (42 male and 24 female) who met the inclusion criteria were identified from the database. The mean age at endoscopic procedure was 56.2 years (range: $33-82$ years).

One patient underwent surgery at $\mathrm{L} 2 / 3,4$ patients at L3/4, 32 patients at L4/5, and 29 patients at L5/S1 . The mean duration of symptoms prior to endoscopic procedure was 19 months (range: 1-240 months). Four patients reported to have symptoms since more than 10 years. Fifty-five procedures were performed using a $19-\mathrm{mm}$ tubular retractor and 11 procedures were performed using a $15-\mathrm{mm}$ tubular retractor. A total of 4 different surgeons were involved the procedures. Three among those were trained by the senior author of the manuscript, who has performed the majority of cases. Each surgeon had substantial ( $>1000$ procedures) experience in microsurgical lumbar spine surgery.

Intraoperatively in one case a switch from a 15$\mathrm{mm}$ to a $19-\mathrm{mm}$ tubular retractor was performed due to limited instrument angulation caused by obesity. In 60 cases adhesive epidural tissue that limited the mobility of the exiting nerve root and thecal sac was identified intraoperatively.

In 20 cases DA was performed and in 46 cases DPD was performed; a detailed compilation of the 2 groups with respect to patient characteristics, MRI findings, and clinical outcome is shown on Table 2.

There was no conversion to open surgery and no technical complication occurred with the endoscopic system. The mean surgical time was 61 minutes (range: 25-129 minutes). An incidental dural tear was noted in seven cases $(10.6 \%)$. In all cases the dural tear was repaired using an autologous muscle graft and a fibrin-sealed collagen sponge and all patients were mobilized on the day of surgery. ${ }^{31}$

\section{Clinical Findings: Preoperative, Postoperative, and Final Follow-Up}

\section{Preoperative}

The primary indication for surgical treatment was failure of conservative treatment for radiculopathy, which was the cause for restricted quality of life.

Fifty-six patients had radicular leg pain $(84.8 \%)$, the mean pain intensity was 6 on the numeric rating scale (NRS; range: 1-10). In 34 patients a paresis $(51.5 \%)$ was documented. Fifty-four patients had back pain $(81.8 \%)$ with a mean intensity of 6 on the NRS (range: 2-10) and among those 4 patients had a history of chronic back pain (more than 10 years). The mean ODI was $24.2 \%$ (range: $0 \%-46 \%$ ).

The degree of paresis was $4 / 5$ in 17 patients, $3 / 5$ in 15 patients, and $2 / 5$ in 2 patients. In 25 patients a sensory deficit $(37.8 \%)$ was noted. 
Table 2. Patient characteristics, MRI findings, and clinical outcome.

\begin{tabular}{lll}
\hline & Decompression Alone & Decompression and Partial Discectomy \\
\hline No. of patients & 20 & 46 \\
Male, n (\%) & $10(50)$ & $32(69)$ \\
Age at surgery, mean (range), y & $58.3(25-81)$ & $55.3(33-82)$ \\
MRI findings & 12 & 4 \\
Group 1 & 5 & 14 \\
Group 2 & 3 & 9 \\
Group 3 & n.a. & 15 \\
Group 4 & 17 & 15 \\
Facet hypertrophy & 4 & 7 \\
Facet joint effusion & n.a. & n.a. \\
Disc degeneration grade 1 & 1 & 7 \\
Disc degeneration grade 2 & 2 & 27 \\
Disc degeneration grade 3 & 11 & 11 \\
Disc degeneration grade 4 & 6 & 92.8 \\
Disc degeneration grade 5 & 66.7 & 0.7 \\
Clinical success at follow-up, \% & 1.3 & 16 \\
Mean leg pain at follow-up (NRS) & 20 & 4 \\
Mean ODI at follow-up, \% & 3 & Decompression of contralateral LRS \\
Number of patients with repeat procedure & TLIF & PELD at index segment \\
Surgical technique at repeat procedure & Decompression of LSS & Microsurgical sequestrectomy (2 times) \\
\end{tabular}

Abbreviations: LRS, lateral recess stenosis; LSS, lumbar spinal stenosis; MRI, magnetic resonance imaging; n.a., not applicable; NRS, numeric rating scale; ODI, Oswestry disability index; PELD, percutaneous endoscopic lumbar discectomy; TLIF, transforaminal lumbar interbody fusion.

\section{Postoperative}

All patients were mobilized at the day of surgery. The median hospitalization was 4 days. At discharge 28 patients reported to be free of leg pain $(42.4 \%)$, 37 patients reported an improvement of their leg pain $(56.1 \%)$, and 1 patient reported a worsening of his preoperative leg pain. The mean leg pain intensity improved to 1.4 (range: $0-8$ ) on the NRS. Forty-two patients reported an improvement of back pain $(63.6 \%)$, while 22 patients reported to be free of back pain $(33.3 \%)$, and 2 patient reported unchanged back pain. The mean back pain intensity on the NRS was 1.8 (range: $0-9$ ), and mean ODI improved to $10.2 \%$ (range: $0 \%-36 \%$ ).

At discharge, in 45 patients full motor strength $(68.2 \%)$ was documented, in 19 patients an improvement of motor strength was noted $(28.8 \%)$, and in 2 patients no improvement was noted (3.0\%).

No postoperative wound healing problems or wound infection were noted. None of the 7 patients with intraoperative dural tear reported any kind of discomfort which could be related to the cerebrospinal fluid leak or development of a pseudomeningocele.

\section{Final Follow-Up}

The mean follow-up was 45.0 months (range: 16-86 months) at which 57 patients $(86.4 \%$ follow-up rate) were evaluated. Nine patients $(13.6 \%)$ were lost to final follow-up evaluation. Two patients died due to causes unrelated to endoscopic procedure 11 and 26 months after the procedure. Seven patients could not be reached by telephone.

Fifty-two patients reported to be free of leg pain $(91.1 \%)$; the mean leg pain intensity on the NRS was 0.9 (range: $0-7$ ). Forty-two patients reported to be free noticeable back pain $(73.7 \%)$; the mean back pain intensity was 2.0 (range: $0-8$ ), and the mean ODI was $16 \%$ (range: $0 \%-60 \%$ ). Forty-nine patients had full muscle strength (85.9\%), and 48 patients reported to have no sensory disturbance $(84.2 \%)$.

According to the MacNab criteria 49 patients had excellent or good results $(85.9 \%)$ and 50 patients reported to have no limitation in their daily activity $(87.7 \%)$. Forty-four patients reported to participate in sports activity regularly $(77.2 \%) ; 4$ patients reported the daily intake of pain medication $(7.0 \%)$.

\section{Repeat Procedure}

In 7 cases $(12.2 \%)$ a repeat procedure was performed. Those procedures were related to either recurrent disc herniation or progress of degenerative changes with delayed instability in 1 case $(1.7 \%)$.

(1) One patient underwent transforaminal lumbar interbody fusion (TLIF) due to the increased mechanical low back pain 6 months after initial endoscopic procedure

(2) One patient developed radicular pain on the contralateral side and decompression was 
performed at the same level for LRS 3 years after the initial procedure.

(3) One patient underwent microsurgical decompression of central LSS in the adjacent segment 16 months after the initial procedure.

(4) One patient developed radicular pain caused by a disc herniation at the previously decompressed (DA) level and underwent endoscopic discectomy 2 years after the initial procedure.

(5) One patient underwent a percutaneous endoscopic lumbar discectomy for resection of a recurrent extraforaminal disc herniation 6 years after the initial procedure.

(6) One patient underwent microsurgical resection of a recurrent disc herniation 11 months after the initial procedure.

(7) One patient underwent microsurgical resection of a recurrent disc herniation 4 months after the initial procedure.

\section{Preoperative MRI Evaluation}

According to the preoperative MRI scan 16 patients were assigned to group 1, 19 patients were assigned to group 2, 14 patients were assigned to group 3, and 17 patients were assigned to group 4 . Hypertrophy of the facet joint with compression of the spinal canal and dura was noted in 45 cases. Facet joint effusion on the affected side was noted in 14 patients.

Disc degeneration of grade 2 according to Pfirrmann et $\mathrm{al}^{30}$ was noted in 2 patients, of grade 3 in 9 patients, of grade 4 in 39 patients, and of grade 5 in 16 patients.

The rate of clinical success according to MacNab in group 1 was $73 \%$, in group 2 it was $79 \%$, and in groups 3 and 4 it was $100 \%$.

\section{Statistical Analysis}

Postoperative results were as follows:

(1) Mean leg pain intensity was 1.3 in the DPD and 1.6 in the DA group, which was not significantly different $(P=.483)$.

(2) Mean back pain intensity was 1.7 in the DPD and 1.6 in the DA group, which was not significantly different $(P=.400)$.

(3) Mean ODI was 20.2 in the DPD and 17.8 in the DA group, which was not significantly different $(P=.480)$.
Final follow-up results were as follows:

(1) Mean leg pain intensity was 0.7 in the DPD and 1.3 in the DA group, which was not significantly different $(P=.118)$.

(2) Mean back pain intensity was 1.5 in the DPD and 2.6 in the DA group, which was not significantly different $(P=.115)$.

(3) Mean ODI was 16.0 in the DPD and 20.0 in the DA group, which was not significantly different $(P=.258)$.

(4) The clinical success rate was $92 \%$ in the DPD group and $67 \%$ in the DA group, which was not significantly different (Fisher exact test, $P$ $=.024)$.

(5) There was no statistically significant difference in the rate of repeated procedure between the DPD and DA groups (Fisher exact test, $P=.365$ ).

\section{DISCUSSION}

LSS is a disorder that emerges at the end of a natural degenerative process. The degeneration of the disc with loss of disc height, consecutive hypertrophy of the facet joints, and thickening of the ligamentum flavum are frequently observed on patients over the age of 65 years. ${ }^{32}$ The nerve root passes the lateral recess and any degenerative disorder such as disc bulge, disc protrusion, disc herniation, facet joint hypertrophy, synovial facet joint, or hypertrophy of the ligamentum flavum can cause as stenosis of the lateral recess and a compression of the nerve root. ${ }^{32-34}$ In the past decades, spine surgeons had the ambition to get a better understanding of how to perform decompression of lumbar canal stenosis and satisfactory results for various surgical techniques have been reported. ${ }^{20,35-38}$ Even though the actual technique for decompression might have a limited influence on the long-term clinical outcome, there are 2 aspects which have become a focus for decompression of LSS and LRS.

These aspects are the surgical approach and the amount of facet joint resection. Minimally invasive techniques have been developed to preserve the posterior elements with the approach and reduce trauma to muscle and soft tissue. This results in lower levels of systemic inflammatory parameters, lower postoperative pain levels requiring less intake of analgesics, earlier mobilization, and faster return to work. ${ }^{15,16,19,39-45}$ Basically there are 2 different 
endoscopic techniques, the so-called "full endoscopic" technique, which is performed under continuous irrigation in single-handed fashion using special instruments, ${ }^{46,47}$ and the tubular-assisted "microendoscopic" technique, which is performed in bimanual fashion using standard microsurgical instruments. $^{15,48}$ The spectrum of indications for full endoscopic techniques has enlarged from discectomy towards the treatment of LSS and LRS due to technical advancements in optics, instruments, and drills, and this particular surgery might be performed via a contralateral, interlaminar, or transforaminal approach..$^{8,9,47,49,50}$ A significant reduction in leg pain as well as clinical success rates ranging from $75.0 \%$ to $92.3 \%$ depending on the approach and the event of previous surgery have been reported for full endoscopic procedures. ${ }^{8-11,22,47,49-60}$

However, one major concern regarding the full endoscopic technique is the prolonged learning curve and the limited option to manage intraoperative complication such as dural tears or dissection of adhesive epidural tissue. ${ }^{49,61-63}$ In contrast to the full endoscopic technique the microendoscopic decompression of LRS is solely performed via an interlaminar approach. The application of microsurgical instruments allows for decompression of the nerve root, dissection of adhesive epidural tissue, and partial discectomy, if necessary. A very steep learning curve has been reported for the microendoscopic technique, which has been used in the present study. ${ }^{64}$ However, with the microendoscopic technique, endoscopic dural repair can be performed without conversion to open surgery and the number of procedures to reach the asymptote is smaller compared to full endoscopic techniques. $^{26,64,65}$ The interlaminar approach risks harming the facet joints. To prevent the progression of postoperative spinal instability and achieve successful clinical outcome it is recommended to preserve the facet joints as much as possible. ${ }^{66-68} \mathrm{It}$ has been reported that preservation of the ipsilateral facet joints is difficult to achieve via a unilateral approach. A reduction rate of facet size from $6 \%$ to $22.6 \%$ has been reported. ${ }^{40,69,70}$ Therefore some authors recommend performing a contralateral approach for decompression of LRS. Several advantages have been stated, such as sparing of the facet joint, better visualization of the neuroforamen, and undercutting of the contralateral ligamentum flavum and superior articular process. $^{9,58,71,72}$

Even though no postoperative computed tomography or MRI scan was performed regularly in the present study the review of the endoscopic video material revealed that resection of the medial third up to two-thirds of the facet joint was indeed performed to perform adequate decompression. Some authors believe that ipsilateral decompression and resection of the facet joint might accelerate the spinal deformity and leads to instability. ${ }^{70,73}$ In the present series there was 1 patient with a long history of chronic low back pain $(1.7 \%)$ who underwent a TLIF procedure due to increased mechanical low back pain. It should be stated that the DA in this patients was performed due to the onset of a footdrop paresis and that this patient initially refused to undergo the TLIF procedure. In addition of all the aforementioned approach-related advantages, the authors also would like mention some limitations. At first the dissection and resection of adhesive epidural tissue, which is often located ventrolateral to the origin of the nerve root and the underlying disc space, is limited via a contralateral and transforaminal approach. Furthermore, the feasibility to perform a partial discectomy and to dissect the dura and the exiting nerve root of the osseous structures and the disc space is limited. Finally, the distance from the skin level to the lateral recess via a contralateral approach is longer compared to the ipsilateral approach and therefore the instrument handling might be limited, especially if it comes to difficult steps of the procedure such as dissection of scar tissue and repair or closure of a dural tear.

The rate of clinical success following contralateral approach has been reported to vary from $92 \%$ to $95 \%$ within a mean follow-up of about 13 months ${ }^{58,71}$; a success rate of $92 \%$ has been reported for an ipsilateral transforaminal approach with a mean follow-up of 34 months, ${ }^{74}$ which is higher compared to the overall clinical success of $86.6 \%$ of the present study. However, the follow-up of the present study was longer compared to the aforementioned studies.

The number of studies which assessed the clinical outcome after tubular or endoscopic decompression of LRS is limited. The ODI of the present study (ie $16 \%$ ) is in range of the ODI values reported in the literature review: $10 \%$ to $25 \%$. This indicates that partial resection of the ipsilateral facet joint via a 
tubular approach has no significant influence on the ODI at follow-up.

It has been reported that stand-alone decompression is effective for the treatment of LRS caused by calcified disc herniation. The rate of clinical success has been reported to be up to $90.9 \%$ with 5 years of follow-up and none of the patients with stand-alone decompression required a revision procedure. ${ }^{75}$ This success rate is considerably higher compared to the rate of $66.7 \%$ in the present study. In contrast, in the present study $92 \%$ of patients who underwent decompression and partial discectomy reported clinical success according to MacNab criteria. However, other patient-reported outcome parameters such as leg pain, back pain, and ODI did not show a statistically significant difference.

Furthermore, it is difficult to identify which aspect might have caused this difference in clinical outcome. In the authors' experience sufficient decompression of the nerve root is the key for clinical success. In the present series the mean duration of clinical symptoms was 19 months. The presence of epidural adhesive tissue with consecutive fixation of the nerve root is frequently observed in patients with a long duration of preoperative symptoms. The origin of this tissue is mainly caused by the resorption process of disc fragments or disc protrusion. The endoscopic visualization allows for ideal illumination of the surgical field as well as identification and differentiation of the dura, the disc, and adhesive tissue. ${ }^{27}$ In cases with previously diagnosed subligamentous disc protrusion it might be difficult to sufficiently decompress and mobilize the nerve root via decompression alone. In those cases, partial discectomy was performed to completely release the dura and the origin of the nerve root at the level of the disc space. If necessary, this dissection should be continued into the neuroforamen. Some spinal surgeons might argue that such aggressive decompression might result in acceleration of spinal instability or recurrent disc herniation and an increased repeated procedure rate. The MRI evaluation revealed that $83 \%$ of discs showed progressive degeneration prior to endoscopic procedure, which might have an impact on the development of recurrent disc herniation as well. Three among those patients who underwent DPD required a revision procedure for recurrent disc herniation $(6.8 \%)$ and none of those patients developed mechanical low back pain. Therefore, the authors believe that it is worth performing extensive decompression and mobilization of the nerve root including a partial discectomy if necessary.

\section{Limitations}

There are limitations to this study which necessitate discussion. It is not possible to control the subtleties of surgeon-driven patient selection and intraoperative technique. It has been reported that based on MRI assessment studies the foraminal area and foraminal diameter significantly changes from supine to upright position. ${ }^{76}$ It is therefore conceivable that nerve root compression might change in prone position, which could influence the intraoperative decision process for performing DPD or DA alone. All surgeons involved in the present study had substantial experience in microsurgical lumbar spine surgery and all were trained at the senior author's institution. We assume that the bias of surgical experience had minimal influence on the results of the present study. However, there is no reliable score to objectively evaluate the mobility of the nerve root and amount of nerve root decompression that can be applied intraoperatively. Therefore, each surgeon might assess the mobility of the nerve root, which is the key factor in this study, slightly differently. In addition to these potential confounders there was also observed variability in follow-up times for each patient. The results demonstrated that ODI increased over time, as well as intensity of leg and back pain changes. In order to overcome some of these limitations, further prospective and potentially randomized studies with a larger cohort will be needed.

\section{CONCLUSION}

The results of the present study demonstrate that endoscopic decompression of LRS is a safe technique and offers a high rate of clinical success. DPD achieves a clinical success rate of up to $92 \%$. In the present series of patients, the rate of recurrent disc herniation was $6.8 \%$ and the rate of delayed spinal instability was $1.7 \%$ within 45 months.

\section{REFERENCES}

1. Kalichman L, Cole R, Kim DH, et al. Spinal stenosis prevalence and association with symptoms: the Framingham Study. Spine J. 2009;9(7):545-550.

2. Szpalski M, Gunzburg R. Lumbar spinal stenosis in the elderly: an overview. Eur Spine J. 2003;12(suppl 2):S170-175.

3. Taylor VM, Deyo RA, Cherkin DC, Kreuter W. Low back pain hospitalization. Recent United States trends and regional variations. Spine. 1994;19(11):1207-1212; discussion 1213.

4. Lee CK, Rauschning W, Glenn W. Lateral lumbar spinal 
canal stenosis: classification, pathologic anatomy and surgical decompression. Spine. 1988;13(3):313-320.

5. Ciric I, Mikhael MA, Tarkington JA, Vick NA. The lateral recess syndrome. A variant of spinal stenosis. J Neurosurg. 1980;53(4):433-443.

6. Weinstein JN, Tosteson TD, Lurie JD, et al. Surgical versus nonsurgical therapy for lumbar spinal stenosis. $N$ Engl $J$ Med. 2008;358(8):794-810.

7. Lurie J, Tomkins-Lane C. Management of lumbar spinal stenosis. BMJ. 2016;352:h6234.

8. Birjandian Z, Emerson S, Telfeian AE, Hofstetter CP. Interlaminar endoscopic lateral recess decompression - surgical technique and early clinical results. J Spine Surg. 2017;3(2):123132.

9. Krzok G, Telfeian AE, Wagner R, Hofstetter CP, Iprenburg M. Contralateral facet-sparing sublaminar endoscopic foraminotomy for the treatment of lumbar lateral recess stenosis: technical note. J Spine Surg. 2017;3(2):260-266.

10. Lewandrowski KU. Readmissions after outpatient transforaminal decompression for lumbar foraminal and lateral recess stenosis. Int J Spine Surg. 2018;12(3):342-351.

11. Lewandrowski KU. Endoscopic transforaminal and lateral recess decompression after previous spinal surgery. Int J Spine Surg. 2018;12(2):98-111.

12. Alimi M, Hofstetter CP, Torres-Campa JM, et al. Unilateral tubular approach for bilateral laminotomy: effect on ipsilateral and contralateral buttock and leg pain. Eur Spine J. 2017;26(2):389-396.

13. Katz JN, Lipson SJ, Larson MG, McInnes JM, Fossel $\mathrm{AH}$, Liang $\mathrm{MH}$. The outcome of decompressive laminectomy for degenerative lumbar stenosis. J Bone Joint Surg Am. 1991;73(6):809-816.

14. Mayer HM, Brock M. Percutaneous endoscopic discectomy: surgical technique and preliminary results compared to microsurgical discectomy. J Neurosurg. 1993;78(2) :216-225.

15. Khoo LT, Fessler RG. Microendoscopic decompressive laminotomy for the treatment of lumbar stenosis. Neurosurgery. 2002;51(suppl 5):S146-154.

16. Oertel JM, Burkhardt BW. Endoscopic surgical treatment of lumbar synovial cyst: detailed account of surgical technique and report of 11 consecutive patients. World Neurosurg. 2017;103:122-132.

17. Oertel JM, Mondorf Y, Gaab MR. A new endoscopic spine system: the first results with "Easy GO." Acta Neurochir (Wien). 2009;151(9):1027-1033.

18. Sandhu FA, Santiago P, Fessler RG, Palmer S. Minimally invasive surgical treatment of lumbar synovial cysts. Neurosurgery. 2004;54(1):107-111; discussion 111-112.

19. Oertel JM, Burkhardt BW. Endoscopic intralaminar approach for the treatment of lumbar disc herniation. World Neurosurg. 2017;103:410-418.

20. Minamide A, Yoshida M, Yamada $\mathrm{H}$, et al. Clinical outcomes after microendoscopic laminotomy for lumbar spinal stenosis: a 5-year follow-up study. Eur Spine J. 2015;24(2):396403.

21. Ruetten S, Komp M, Godolias G. A new full-endoscopic technique for the interlaminar operation of lumbar disc herniations using 6-mm endoscopes: prospective 2-year results of 331 patients. Minim Invasive Neurosurg. 2006;49(2):80-87.

22. Ruetten S, Komp M, Merk H, Godolias G. Surgical treatment for lumbar lateral recess stenosis with the fullendoscopic interlaminar approach versus conventional micro- surgical technique: a prospective, randomized, controlled study. $J$ Neurosurg Spine. 2009;10(5):476-485.

23. Ruetten S, Komp M, Merk H, Godolias G. Fullendoscopic interlaminar and transforaminal lumbar discectomy versus conventional microsurgical technique: a prospective, randomized, controlled study. Spine. 2008;33(9):931-939.

24. Fairbank JC, Couper J, Davies JB, O'Brien JP. The Oswestry low back pain disability questionnaire. Physiotherapy. 1980;66(8):271-273.

25. MacNab I. Negative disc exploration. An analysis of the causes of nerve-root involvement in sixty-eight patients. $J$ Bone Joint Surg Am. 1971;53(5):891-903.

26. Burkhardt BW, Oertel JM. Endoscopic spinal surgery using a new tubular retractor with $15 \mathrm{~mm}$ outer diameter. $\mathrm{Br} J$ Neurosurg. 2019; 33(5): 514-521.

27. Burkhardt BW, Wilmes M, Sharif S, Oertel JM. The visualization of the surgical field in tubular assisted spine surgery: is there a difference between HD-endoscopy and microscopy? Clin Neurol Neurosurg. 2017;158:5-11.

28. Carragee EJ, Kim DH. A prospective analysis of magnetic resonance imaging findings in patients with sciatica and lumbar disc herniation. Correlation of outcomes with disc fragment and canal morphology. Spine. 1997;22(14):1650-1660.

29. Fardon DF. Nomenclature and classification of lumbar disc pathology. Spine. 2001;26(5):461-462.

30. Pfirrmann CW, Metzdorf A, Zanetti M, Hodler J, Boos $\mathrm{N}$. Magnetic resonance classification of lumbar intervertebral disc degeneration. Spine. 2001;26(17):1873-1878.

31. Oertel JM, Burkhardt BW. Full endoscopic treatment of dural tears in lumbar spine surgery. Eur Spine $J$. 2017;26(10):2496-2503.

32. Postacchini F. Management of lumbar spinal stenosis. $J$ Bone Joint Surg Br. 1996;78(1):154-164.

33. Siebert E, Pruss H, Klingebiel R, Failli V, Einhaupl KM, Schwab JM. Lumbar spinal stenosis: syndrome, diagnostics and treatment. Nat Rev Neurol. 2009;5(7):392-403.

34. Genevay S, Atlas SJ. Lumbar spinal stenosis. Best Pract Res Clin Rheumatol. 2010;24(2):253-265.

35. Thome C, Zevgaridis D, Leheta O, et al. Outcome after less-invasive decompression of lumbar spinal stenosis: a randomized comparison of unilateral laminotomy, bilateral laminotomy, and laminectomy. $J$ Neurosurg Spine. 2005;3(2):129-141.

36. Orpen NM, Corner JA, Shetty RR, Marshall R. Microdecompression for lumbar spinal stenosis: the early outcome using a modified surgical technique. J Bone Joint Surg Br. 2010;92(4):550-554.

37. den Boogert HF, Keers JC, Marinus Oterdoom DL, Kuijlen JM. Bilateral versus unilateral interlaminar approach for bilateral decompression in patients with single-level degenerative lumbar spinal stenosis: a multicenter retrospective study of 175 patients on postoperative pain, functional disability, and patient satisfaction. J Neurosurg Spine. 2015;23(3):326-335.

38. Fu YS, Zeng BF, Xu JG. Long-term outcomes of two different decompressive techniques for lumbar spinal stenosis. Spine. 2008;33(5):514-518.

39. Huang TJ, Hsu RW, Li YY, Cheng CC. Less systemic cytokine response in patients following microendoscopic versus open lumbar discectomy. J Orthop Res. 2005;23(2):406-411.

40. Yoshimoto M, Miyakawa T, Takebayashi T, et al. Microendoscopy-assisted muscle-preserving interlaminar decompression for lumbar spinal stenosis: clinical results of 
consecutive 105 cases with more than 3-year follow-up. Spine. 2014;39(5):E318-325.

41. Bresnahan L, Ogden AT, Natarajan RN, Fessler RG. A biomechanical evaluation of graded posterior element removal for treatment of lumbar stenosis: comparison of a minimally invasive approach with two standard laminectomy techniques. Spine. 2009;34(1):17-23.

42. Bresnahan LE, Smith JS, Ogden AT, et al. Assessment of paraspinal muscle cross-sectional area following lumbar decompression: minimally invasive versus open approaches. J Clin Spine Surg. 2017;30(3): 162-168.

43. O’Toole JE, Eichholz KM, Fessler RG. Surgical site infection rates after minimally invasive spinal surgery. $J$ Neurosurg Spine. 2009;11(4):471-476.

44. Xu BS, QST, Xia Q, Ji N, Hu YC. Bilateral decompression via unilateral fenestration using mobile microendoscopic discectomy technique for lumbar spinal stenosis. Orthop Surg. 2010;2(2):106-110.

45. He J, Xiao S, Wu Z, Yuan Z. Microendoscopic discectomy versus open discectomy for lumbar disc herniation: a meta-analysis. Eur Spine J. 2016;25(5):1373-1381.

46. Kambin P. Percutaneous endoscopic discectomy. $J$ Neurosurg. 1993;79(6):968-969; author reply 969-970.

47. Kambin P, Casey K, O;Brien E, Zhou L. Transforaminal arthroscopic decompression of lateral recess stenosis. $J$ Neurosurg. 1996;84(3):462-467.

48. Foley KT, Smith MM. Microendoscopic discectomy. Tech Neurosurg. 1997;3:301-307.

49. Ruetten S, Komp M, Hahn P, Oezdemir S. Decompression of lumbar lateral spinal stenosis: full-endoscopic, interlaminar technique [in German. Oper Orthop Traumatol. 2013;25(1):31-46.

50. Tang S, Jin S, Liao X, Huang $\mathrm{K}$, Luo J, Zhu $\mathrm{T}$. Transforaminal percutaneous endoscopic lumbar decompression by using rigid bendable burr for lumbar lateral recess stenosis: technique and clinical outcome. Biomed Res Int. 2018;2018:2601232.

51. Chen X, Qin R, Hao J, et al. Percutaneous endoscopic decompression via transforaminal approach for lumbar lateral recess stenosis in geriatric patients. Int Orthop. 2019;43(5):12631269.

52. Kapetanakis S, Gkantsinikoudis N, Papathanasiou JV, Charitoudis G, Thomaidis T. Percutaneous endoscopic ventral facetectomy: an innovative substitute of open decompression surgery for lateral recess stenosis surgical treatment? $J$ Craniovertebr Junction Spine. 2018;9(3):188-195.

53. Kapetanakis S, Gkantsinikoudis $\mathrm{N}$, Thomaidis $\mathrm{T}$, Charitoudis G, Theodosiadis $\mathrm{P}$. The role of percutaneous transforaminal endoscopic surgery in lateral recess stenosis in elderly patients. Asian Spine J. 2019;13(4):638-647.

54. Ahn Y, Keum HJ, Lee SG, Lee SW. Transforaminal endoscopic decompression for lumbar lateral recess stenosis: an advanced surgical technique and clinical outcomes. World Neurosurg. 2019;125:916-924.

55. Lewandrowski KU. "Outside-in" technique, clinical results, and indications with transforaminal lumbar endoscopic surgery: a retrospective study on 220 patients on applied radiographic classification of foraminal spinal stenosis. Int $J$ Spine Surg. 2014;1;8;26.

56. Shin SH, Bae JS, Lee SH, Keum HJ, Kim HJ, Jang WS. Transforaminal endoscopic decompression for lumbar spinal stenosis: a novel surgical technique and clinical outcomes. World Neurosurg. 2018;114:e873-e882.

57. Hwang JH, Park WM, Park CW. Contralateral interlaminar keyhole percutaneous endoscopic lumbar surgery in patients with unilateral radiculopathy. World Neurosurg. 2017;101:33-41

58. Kim HS, Patel R, Paudel B, et al. Early outcomes of endoscopic contralateral foraminal and lateral recess decompression via an interlaminar approach in patients with unilateral radiculopathy from unilateral foraminal stenosis. World Neurosurg. 2017;108:763-773.

59. Li ZZ, Hou SX, Shang WL, Cao Z, Zhao HL. Percutaneous lumbar foraminoplasty and percutaneous endoscopic lumbar decompression for lateral recess stenosis through transforaminal approach: technique notes and 2 years followup. Clin Neurol Neurosurg. 2016;143:90-94.

60. Wang YP, Zhang W, Li BL, Sun YP, Ding WY, Shen Y. Suprapedicular foraminal endoscopic approach to lumbar lateral recess decompression surgery to treat degenerative lumbar spinal stenosis. Med Sci Monit. 2016;22:4604-4611.

61. Hsu HT, Chang SJ, Yang SS, Chai CL. Learning curve of full-endoscopic lumbar discectomy. Eur Spine $J$. 2013;22(4):727-733.

62. Morgenstern R, Morgenstern C, Yeung AT. The learning curve in foraminal endoscopic discectomy: experience needed to achieve a $90 \%$ success rate. SAS J. 2007;1(3):100 107.

63. Lee CH, Choi M, Ryu DS, et al. Efficacy and safety of full-endoscopic decompression via interlaminar approach for central or lateral recess spinal stenosis of the lumbar spine: a meta-analysis. Spine. 2018;43(24):1756-1764.

64. Burkhardt BW, Oertel JM. The learning process of endoscopic spinal surgery for degenerative cervical and lumbar disorders using the EasyGO! system. World Neurosurg. 2018;119:479-487.

65. Oertel JM, Burkhardt BW. Full endoscopic treatment of dural tears in lumbar spine surgery. Eur Spine J. 2017, 26(10):2496-2503.

66. Abumi K, Panjabi MM, Kramer KM, Duranceau J, Oxland T, Crisco JJ. Biomechanical evaluation of lumbar spinal stability after graded facetectomies. Spine. 1990;15(11):1142-1147.

67. Haher TR, O’Brien M, Dryer JW, Nucci R, Zipnick R, Leone DJ. The role of the lumbar facet joints in spinal stability. Identification of alternative paths of loading. Spine. 1994;19(23):2667-2670 discussion 2671.

68. Zander T, Rohlmann A, Klockner C, Bergmann G. Influence of graded facetectomy and laminectomy on spinal biomechanics. Eur Spine J. 2003;12(4):427-434.

69. Ikuta K, Arima J, Tanaka T, et al. Short-term results of microendoscopic posterior decompression for lumbar spinal stenosis. Technical note. J Neurosurg Spine. 2005;2(5):624-633.

70. Matsumura A, Namikawa $T$, Terai $H$, et al. The influence of approach side on facet preservation in microscopic bilateral decompression via a unilateral approach for degenerative lumbar scoliosis. Clinical article. J Neurosurg Spine. 2010;13(6):758-765.

71. Alimi M, Njoku I, Jr., Cong GT, et al. Minimally invasive foraminotomy through tubular retractors via a contralateral approach in patients with unilateral radiculopathy. Neurosurgery. 2014;10(suppl 3):436-447; discussion 446- 447.

72. Ogrenci A, Koban O, Yaman O, Yilmaz M, Dalbayrak S. Clinical comparison between patients operated for unilateral 
radiculopathy via a contralateral (facet-sparing) and ipsilateral side approach. Turk Neurosurg. 2018;28(4):610-615.

73. Martin BI, Mirza SK, Comstock BA, Gray DT, Kreuter W, Deyo RA. Reoperation rates following lumbar spine surgery and the influence of spinal fusion procedures. Spine. 2007;32(3):382-387.

74. Lewandrowski KU, De Carvalho PST, De Carvalho P, Yeung A. Minimal clinically important difference in patientreported outcome measures with the transforaminal endoscopic decompression for lateral recess and foraminal stenosis. Int $J$ Spine Surg. 2020;14(2):254-266.

75. Kulkarni AG, Patel R, Dutta S, Patil V. Stand-alone lateral recess decompression without discectomy in patients presenting with claudicant radicular pain and MRI evidence of lumbar disc herniation: a prospective study. Spine. 2017;42(13):984-991.

76. Lang G, Vicari M, Siller A, et al. Preoperative assessment of neural elements in lumbar spinal stenosis by upright magnetic resonance imaging: an implication for routine practice? Cureus. 2018;10(4):e2440.

Disclosures and COI: The authors declare that they have no conflict of interest. J. Oertel acts as a consultant for the Karl Storz company.This research did not receive any specific grant from funding agencies in the public, commercial, or notfor-profit sectors.

Corresponding Author: Joachim Oertel, MD, Klinik für Neurochirurgie, Universitätsklinikum des Saarlandes, Kirrbergerstraße 100, Gebäude 90.5, 66424 Homburg, Germany. Phone: +49-6841-1624400; Fax: +49-6841-16-24480; Email: oertelj@ freenet.de.

Published 26 February 2021

This manuscript is generously published free of charge by ISASS, the International Society for the Advancement of Spine Surgery. Copyright @ 2021 ISASS. To see more or order reprints or permissions, see http://ijssurgery.com. 